\title{
OPEN Reduction of mortality by catheter ablation in real-world atrial fibrillation patients with heart failure
}

\author{
Pil-Sung Yang ${ }^{1,3}$, Daehoon Kim ${ }^{2,3}$, Jung-Hoon Sung ${ }^{1}$, Eunsun Jang ${ }^{2}$, Hee Tae Yu², \\ Tae-Hoon Kim², Jae-Sun Uhm², Jong-Youn Kim², Hui-Nam Pak ${ }^{2}$, Moon-Hyoung Lee ${ }^{2} \&$ \\ Boyoung Joung ${ }^{2 \bowtie}$
}

Whether catheter ablation for atrial fibrillation (AF) improves survival and affects other outcomes in real-world heart failure (HF) patients is unclear. This study aimed to evaluate whether ablation reduces death, and other outcomes in real-world AF patients with HF. Among 834,735 patients with AF from 2006 to 2015 in the Korean National Health Insurance Service database, 3173 HF patients underwent AF ablation. Propensity score weighting was used to correct for differences between the groups. During median 54 months follow-up, the risk of all-cause death in ablated patients was less than half of that in patients with medical therapy (2.8 vs. 6.2 per 100 person-years; hazard ratio [HR] $0.42,95 \%$ confidence interval $[\mathrm{Cl}] 0.27-0.65, p<0.001)$. Ablation was related with lower risk of cardiovascular death (HR $0.38,95 \% \mathrm{Cl} 0.32-0.62, p<0.001$ ), HF admission (HR 0.39, 95\% Cl 0.33-0.46, $p<0.001$ ) and stroke/systemic embolism (HR $0.44,95 \% \mathrm{Cl} 0.37-0.53, p<0.001$ ). In subgroup analysis, the risk of all-cause death was reduced in most subgroups except in the elderly ( $\geq 75$ years) and strictly anticoagulated patients. Ablation may be associated with reduced risk of all-cause death and cardiovascular death in real-world AF patients with HF, supporting the role of AF ablation in patients with HF.

Atrial fibrillation (AF) and heart failure are important cardiac conditions associated with the patient's morbidity and mortality ${ }^{1,2}$. The two conditions often coexist and can promote each other. Up to $30 \%$ of patients with heart failure have $\mathrm{AF}^{3-5}$. $\mathrm{AF}$ in patients with heart failure is associated with increased hospitalization, the burden on the health care system, stroke, and mortality ${ }^{6,7}$. Loss of atrial contraction, irregular and rapid ventricular rates in AF can lead to left ventricular dysfunction and decreased cardiac output ${ }^{8,9}$, and these features of AF may be at least partially contributed to poor prognosis of heart failure patients.

Catheter ablation for AF is more effective than antiarrhythmic drugs (AADs) in reducing AF recurrences; AF catheter ablation also extends the duration of the sinus rhythm and improves the patient's quality of life ${ }^{10,11}$. Several observational studies have shown that maintaining sinus rhythm by AF catheter ablation in heart failure patients can significantly improve cardiac function ${ }^{12-14}$. Recently, a trial evaluating ablation compared with medical therapy in symptomatic patients with $\mathrm{AF}$ and heart failure provided evidence suggesting that successful ablation may extend survival ${ }^{15}$. However, because only $13.2 \%$ of the screened heart failure patients were enrolled in randomization in the study, the effect of AF ablation in heart failure patients is still controversial. In the CABANA (Catheter Ablation vs. Antiarrhythmic Drug Therapy for Atrial Fibrillation) trial, the recently performed randomized controlled trial on the effects of AF catheter ablation ${ }^{16}$, AF catheter ablation did not significantly reduce the primary endpoint (a composite of death, disabling stroke, serious bleeding, or cardiac arrest) compared to medical treatment.

Our study aimed to determine whether AF catheter ablation in heart failure patients can reduce the risk of all-cause death, cardiovascular death, and other cardiac events in a real-world nationwide cohort.

\footnotetext{
${ }^{1}$ Department of Cardiology, CHA Bundang Medical Center, CHA University, Seongnam, Republic of Korea. ${ }^{2}$ Division of Cardiology, Department of Internal Medicine, Yonsei University College of Medicine, 50-1 Yonsei-ro, Seodaemun-gu, Seoul 03722, Republic of Korea. ${ }^{3}$ These authors contributed equally: Pil-Sung Yang and Daehoon Kim. ${ }^{\square}$ email: cby6908@yuhs.ac
} 


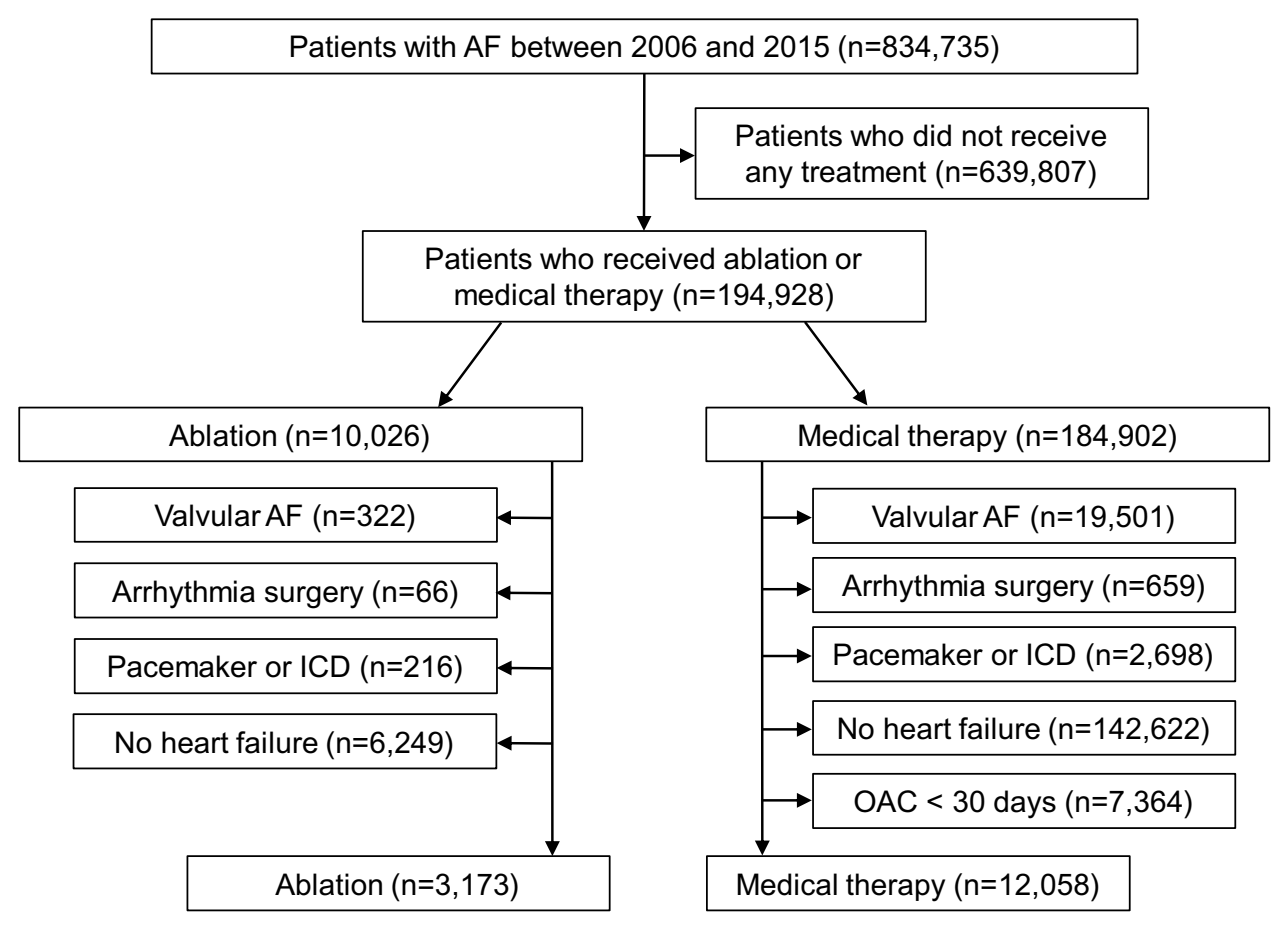

Figure 1. Flowchart of the enrollment and analysis of the study population. AF, atrial fibrillation; ICD, implantable cardioverter-defibrillator; OAC, oral anticoagulant.

\section{Methods}

This study was based on the national health claims database (NHIS-2016-4-009) established by the National Health Insurance Service (NHIS) of Korea. The NHIS is the single insurer managed by the Korean government. The majority $(97.1 \%)$ of Korean citizens are mandatory subscribers to the NHIS, and the remaining $3 \%$ of the population is under the Medical Aid program. As the NHIS database contains the information of Medical Aid users, the database can be considered to be representative of the entire Korean population ${ }^{3-5}$. All pertinent data including patients' sociodemographic information, use of inpatient and outpatient services, pharmacy-dispensing claims, and mortality can be accessed through this database. The NHIS also runs a regular health check-up program for all citizens. NHIS subscribers are recommended to undergo check-ups at least biennially, and the check-up includes blood tests, chest X-ray, physical examinations, and questionnaires for medical history.

The present study was approved by the Institutional Review Board of Yonsei University Health System (4-2016-0179). The board waived the condition of obtaining informed consent for study participation. All methods included in this study were carried out in accordance with relevant guidelines and regulations.

Study population. From January 1, 2006, to December 31, 2015, 834,735 adult patients (18 years old) newly diagnosed with AF were identified in the Korean NHIS database covering a population of 51.5 million. AF was confirmed by the diagnostic code (International Classification of Disease 10th revision [ICD-10] code: I48). Only patients who were diagnosed during hospitalization or diagnosed at least two times in an outpatient clinic were confirmed as AF to ensure the accuracy of the diagnosis. The accuracy of this definition has already been validated in previous studies using the Korean NHIS data. A positive predictive value was $94.1 \%^{3-5,17-19}$. From newly diagnosed AF patients, patients treated with AF catheter ablation or medical therapy (AADs or rate control drugs prescribed for at least 90 days within one year of enrollment) were included in the study population. Catheter ablation for AF was identified using the corresponding Korean NHIS procedure codes for AF catheter ablation (M6542 or M6547) with an admission diagnosis of AF.

The time at risk was counted from the index date of the first AF treatment for both patients with AF ablation and those with medical therapy. The time at risk in patients who underwent AF ablation without prior medical treatment was counted from the date of the first AF ablation. The effect of AF ablation was analyzed as a timevarying exposure. The exclusion criteria were those with mitral stenosis, a history of mitral valve replacement, surgical AF ablation (Maze surgery), or implantation of cardiac implantable electronic device, and those without heart failure history. Patients who had oral anticoagulants less than 30 days during the same period were additionally excluded from the medical therapy group. After exclusions, 3173 ablated patients and 12,058 medically treated patients remained for analysis (Fig. 1).

Covariates. Baseline comorbidities were defined based on inpatient and outpatient clinic diagnosis and prescription drugs prior to the index date. Similar to previous studies using Korean NHIS data, patients diagnosed in admission or confirmed at least twice in an outpatient clinic were considered to have a comorbid (Supplementary Table S1) $)^{3-5,17-19}$. The patient's economic status was determined on the basis of the relative economic level 
classified into 10 tiers according to their health insurance premium of the index year. The use of medication was identified based on prescription claims in the NHIS database within 90 days prior to the index date.

Clinical outcome events and assessments. To assess clinical outcomes, patients were followed until the end of the study period (December 31, 2016) or until death. All-cause death, cardiovascular death, and sudden cardiac death were evaluated. Vital status and the date of death were identified from the data registered in the National Population Registry of the Korea National Statistical Office, based on death certificates and the unique personal identification number ${ }^{3-5,17-19}$. Since the NHIS and National Statistical Office are national organizations covering all Korean citizens, this approach allows complete confirmation of death events.

The definitions of clinical outcomes are presented in Supplementary Table S1. Ischemic stroke was identified by discharge diagnosis (ICD-10 codes: I63, I64) with concomitant brain imaging studies. The accuracy of this definition for ischemic stroke has been validated in previous studies using the Korean NHIS data ${ }^{3-5,17-19}$. It must be noted that only the first event of each outcome was considered in the study when there are multiple events.

To indirectly assess the relationship between successful rhythm control by ablation and outcomes, we considered "Cardioversion or repeated ablation" as an indicator of AF recurrence after ablation. We also performed a validation study of this definition for detecting AF recurrence (Supplement Figure S1).

Statistical methods. Baseline characteristics between patients with AF ablation and those with medical therapy were compared using Student's t-test and chi-square test. A propensity score, the probability of receiving AF ablation, was estimated using logistic regression based on socio-demographics, concomitant diseases, drug use, and duration of AF. (All variables in Table 1 were used). On the basis of the calculated propensity score, inverse probability of treatment weighting was used to balance the differences in baseline characteristics between patients with AF ablation and those with medical therapy. The balance between the two groups was assessed with standardized differences of all baseline covariates, using 0.1 as the threshold representing the imbalance.

Weighted incidence rates were calculated as the weighted number of clinical events during the follow-up period divided by person-years at risk. The $95 \%$ confidence intervals (CI) of incidence rates were estimated by exact Poisson distributions. A weighted log-rank test was used to compare the incidence of mortality and weighted failure curves were plotted. Comparisons between the ablation group and the medical therapy group were analyzed using Cox proportional hazards regressions. The Fine and Gray method was used to regard death as a competing risk when evaluated non-fatal outcomes (i.e. heart failure and stroke/systemic embolism (SE) when evaluated separately $)^{20}$. The proportional hazards assumption was tested based on Schoenfeld residuals ${ }^{21}$.

A two-sided $p$ values of $<0.05$ were considered significant. Data processing and management were performed using SAS version 9.3 (SAS Institute, Cary, NC, USA). Statistical analyses were conducted using R software (version 3.6.1, R Core Team, 2019, Vienna, Austria $)^{22}$.

Sensitivity analyses. First, we conducted subgroup analyses for all-cause death and cardiovascular death stratified by age, sex, heart failure, hypertension, diabetes, vascular disease, $\mathrm{CHA}_{2} \mathrm{DS}_{2}$-VASc score, history of ischemic stroke/transient ischemic attack (TIA), cardioversion, and anticoagulation. Second, instead of using propensity score weighting, we performed an analysis using one-to-one propensity score matching between groups to balance the differences in baseline characteristics. Third, we performed a stratified analysis according to whether medically treated patients were treated with AADs or only with rate control drugs. Fourth, we compared heart failure patients who underwent AF ablation and those who did not have a history of AF. Fifth, we performed "falsification analysis" to determine whether AF ablation was associated with the risks of falsification endpoints such as urinary tract infections, Varicella-zoster, and fall accidents, which should not be associated with AF ablation ${ }^{23}$.

\section{Results}

Differences between ablated and the non-ablated patients. Patients treated with AF catheter ablation had more men, healthier, and higher incomes than those who were medically treated (Table 1). Compared with non-ablated patients, patients receiving ablation were on average 10 years younger and had fewer comorbidities. After propensity score weighting, no significant differences in baseline characteristics were observed between the two groups (Table 1). In multivariable analysis, patients with younger age, higher income, and fewer comorbidities (especially without diabetes, ischemic stroke/TIA, myocardial infarction, and peripheral artery disease) were more likely to undergo AF ablation (Supplementary Table S2).

Patients who underwent AF ablation were younger and healthier than AAD treated (Supplementary Table S3) and rate control patients (Supplementary Table S4). After propensity score weighting, no significant differences in baseline characteristics were observed between the groups.

Reduced all-cause and cardiovascular death in ablated patients. In propensity score-weighted patients, 394 and 3940 all-cause deaths occurred during the median 54 (interquartile ranges: 19, 80) months follow-up period, and weighted annualized rates of all-cause death were 2.8 and 6.2 per 100 person-years in the ablated and medical therapy group, respectively $(p<0.001)$ (Table 2$)$. The cumulative incidence of all-cause death in patients with AF ablation was significantly lower than those with medical therapy $(p<0.001$, Fig. 2A). After fully adjusting the available clinical parameters, the risk of all-cause death was reduced by $58 \%$ in ablated patients compared to patients receiving medical therapy (hazard ratio [HR] $0.42,95 \%$ CI $0.27-0.65, p<0.001$ ) (Table 2). The risk of all-cause mortality was reduced in patients with AF ablation compared to those treated with AAD (HR 0.49, 95\% CI 0.34-0.71, $p<0.001$ ) and rate control only (HR 0.39, 95\% CI 0.27-0.57, $p<0.001$ ) (Table 2). Subgroup analyses found a reduction in the risk of all-cause death in most subgroups, except for the 


\begin{tabular}{|c|c|c|c|c|c|c|}
\hline & Ablation $(\mathrm{N}=3173)$ & $\begin{array}{l}\text { Medical therapy } \\
(\mathrm{N}=12,058)\end{array}$ & SMD (\%) & Ablation $(\mathrm{N}=3173)$ & $\begin{array}{l}\text { Medical therapy } \\
(\mathrm{N}=12,058)\end{array}$ & SMD (\%) \\
\hline \multicolumn{7}{|l|}{ Demographic } \\
\hline Age, years & $60(53,67)$ & $69(62,76)$ & 82.2 & $66(58,74)$ & $66(58,74)$ & 0.9 \\
\hline$<65$ years & $67.4 \%$ & $33.4 \%$ & 72.2 & $42.8 \%$ & $44.2 \%$ & 2.8 \\
\hline 65-75 yearswprk dl & $26.3 \%$ & $32.7 \%$ & 14.1 & $29.9 \%$ & $30.4 \%$ & 1.0 \\
\hline$\geq 75$ years & $4.8 \%$ & $30.3 \%$ & 71.0 & $24.0 \%$ & $22.5 \%$ & 3.5 \\
\hline Male & $71.5 \%$ & $59.1 \%$ & 26.3 & $64.5 \%$ & $62.7 \%$ & 3.7 \\
\hline High income status & $51.7 \%$ & $42.1 \%$ & 19.2 & $49.2 \%$ & $44.3 \%$ & 9.8 \\
\hline AF duration, months & $34.9(11.9,66.8)$ & $23.6(4.6,46.0)$ & 29.6 & $20.1(5.2,49.5)$ & $23.4(3.9,48.7)$ & 5.1 \\
\hline \multicolumn{7}{|l|}{ Risk scores } \\
\hline $\begin{array}{l}\mathrm{CHA}_{2} \mathrm{DS}_{2} \text {-VASc } \\
\text { score }\end{array}$ & $3.0(2.0,5.0)$ & $5.0(3.0,6.0)$ & 73.0 & $4.0(3.0,6.0)$ & $4.0(3.0,6.0)$ & 2.9 \\
\hline mHAS-BLED score ${ }^{*}$ & $3.0(2.0,4.0)$ & $3.0(2.0,4.0)$ & 36.3 & $1.4(0.8,2.7)$ & $3.0(2.1,3.9)$ & 5.3 \\
\hline $\begin{array}{l}\text { Charlson comorbid- } \\
\text { ity index }\end{array}$ & $1.8(0.0,4.8)$ & $3.2(0.0,8.8)$ & 38.7 & $5.0(3.0,7.0)$ & $5.0(3.0,7.0)$ & 1.1 \\
\hline $\begin{array}{l}\begin{array}{l}\text { Hospital frailty risk } \\
\text { score }\end{array} \\
\end{array}$ & $4.0(3.0,6.0)$ & $5.0(3.0,8.0)$ & 62.6 & $2.9(0.7,6.3)$ & $2.3(0.0,7.1)$ & 9.0 \\
\hline \multicolumn{7}{|l|}{ Comorbidities } \\
\hline Heart failure & $100.0 \%$ & $100.0 \%$ & $<0.001$ & $100.0 \%$ & $100.0 \%$ & $<0.1$ \\
\hline Hypertension & $91.3 \%$ & $94.7 \%$ & 13.4 & $93.9 \%$ & $92.7 \%$ & 4.6 \\
\hline Diabetes & $17.8 \%$ & $31.1 \%$ & 31.3 & $27.5 \%$ & $27.0 \%$ & 1.1 \\
\hline Dyslipidemia & $88.5 \%$ & $80.0 \%$ & 23.6 & $83.4 \%$ & $81.5 \%$ & 5.0 \\
\hline Ischemic stroke & $19.9 \%$ & $36.1 \%$ & 36.8 & $32.9 \%$ & $30.7 \%$ & 4.7 \\
\hline TIA & $10.1 \%$ & $11.1 \%$ & 3.4 & $10.0 \%$ & $10.7 \%$ & 2.5 \\
\hline Hemorrhagic stroke & $1.5 \%$ & $3.4 \%$ & 12.7 & $4.8 \%$ & $2.8 \%$ & 10.2 \\
\hline $\begin{array}{l}\begin{array}{l}\text { Myocardial infarc- } \\
\text { tion }\end{array} \\
\end{array}$ & $13.6 \%$ & $21.0 \%$ & 19.7 & $17.3 \%$ & $18.5 \%$ & 3.1 \\
\hline $\begin{array}{l}\text { Peripheral arterial } \\
\text { disease }\end{array}$ & $14.2 \%$ & $18.0 \%$ & 10.3 & $15.1 \%$ & $16.7 \%$ & 4.3 \\
\hline $\begin{array}{l}\text { Chronic kidney } \\
\text { disease }\end{array}$ & $5.9 \%$ & $10.1 \%$ & 15.4 & $11.2 \%$ & $8.8 \%$ & 8.1 \\
\hline \begin{tabular}{|l|}
$\begin{array}{l}\text { End stage renal } \\
\text { disease }\end{array}$ \\
\end{tabular} & $0.9 \%$ & $1.5 \%$ & 5.7 & $1.4 \%$ & $1.3 \%$ & 1.0 \\
\hline Proteinuria & $5.9 \%$ & $6.6 \%$ & 2.9 & $6.5 \%$ & $6.4 \%$ & 0.6 \\
\hline \begin{tabular}{|l|} 
Hyperthyroidism \\
\end{tabular} & $23.2 \%$ & $17.4 \%$ & 14.5 & $17.9 \%$ & $18.4 \%$ & 1.3 \\
\hline Hypothyroidism & $20.6 \%$ & $14.6 \%$ & 15.6 & $13.9 \%$ & $15.5 \%$ & 4.6 \\
\hline Malignancy & $21.9 \%$ & $22.8 \%$ & 2.0 & $18.9 \%$ & $22.1 \%$ & 7.9 \\
\hline COPD & $26.2 \%$ & $38.5 \%$ & 26.7 & $36.9 \%$ & $34.5 \%$ & 5.1 \\
\hline Liver disease & $49.4 \%$ & $43.4 \%$ & 12.0 & $43.7 \%$ & $44.6 \%$ & 1.8 \\
\hline $\begin{array}{l}\text { Hypertrophic cardio- } \\
\text { myopathy }\end{array}$ & $3.3 \%$ & $4.0 \%$ & 3.7 & $3.9 \%$ & $3.8 \%$ & 0.7 \\
\hline History of bleeding & $33.6 \%$ & $33.7 \%$ & 0.3 & $35.9 \%$ & $33.4 \%$ & 5.4 \\
\hline Osteoporosis & $20.9 \%$ & $31.1 \%$ & 23.4 & $26.0 \%$ & $27.9 \%$ & 4.4 \\
\hline Sleep apnea & $2.1 \%$ & $0.5 \%$ & 14.4 & $1.3 \%$ & $0.9 \%$ & 3.8 \\
\hline $\begin{array}{l}\text { Heart failure admis- } \\
\text { sion }\end{array}$ & $7.5 \%$ & $27.2 \%$ & 54.0 & $22.3 \%$ & $22.3 \%$ & $<0.1$ \\
\hline \begin{tabular}{|l|}
$\begin{array}{l}\text { Medication (treat- } \\
\text { ment) }\end{array}$
\end{tabular} & $0.0 \%$ & $0.0 \%$ & & $0.0 \%$ & $0.0 \%$ & \\
\hline OAC & $68.2 \%$ & $76.6 \%$ & 18.9 & $63.3 \%$ & $68.9 \%$ & 12.0 \\
\hline Antiplatelet agents & $79.8 \%$ & $70.6 \%$ & 21.4 & $70.6 \%$ & $71.5 \%$ & 2.0 \\
\hline ACE-inhibitor/ARB & $64.2 \%$ & $74.4 \%$ & 22.2 & $72.5 \%$ & $70.2 \%$ & 5.0 \\
\hline \begin{tabular}{|l|} 
Diuretics \\
\end{tabular} & $53.7 \%$ & $76.7 \%$ & 49.6 & $70.5 \%$ & $69.3 \%$ & 2.7 \\
\hline K sparing diuretics & $17.5 \%$ & $34.8 \%$ & 40.3 & $29.6 \%$ & $29.2 \%$ & 1.0 \\
\hline Statin & $46.9 \%$ & $43.1 \%$ & 7.6 & $45.2 \%$ & $43.1 \%$ & 4.3 \\
\hline Beta blocker & $77.0 \%$ & $65.3 \%$ & 25.9 & $67.1 \%$ & $66.7 \%$ & 0.8 \\
\hline $\begin{array}{l}\text { Dihydropyridine } \\
\text { CCB }\end{array}$ & $34.7 \%$ & $42.5 \%$ & 16.1 & $43.3 \%$ & $40.0 \%$ & 6.7 \\
\hline $\begin{array}{l}\text { Nondihydropyridine } \\
\text { CCB }\end{array}$ & $27.2 \%$ & $20.1 \%$ & 16.6 & $20.9 \%$ & $21.1 \%$ & 0.6 \\
\hline Digoxin & $23.5 \%$ & $45.0 \%$ & 46.6 & $37.6 \%$ & $38.7 \%$ & 2.3 \\
\hline
\end{tabular}


Table 1. Baseline characteristics before and after propensity score weighting. Values are presented as median (Q1, Q3, quartiles [25th and 75th percentiles]) or \%. ${ }^{\star}$ Modified HAS-BLED = hypertension, 1 point: $>65$ years old, 1 point: stroke history, 1 point: bleeding history or predisposition, 1 point: liable international normalized ratio, not assessed: ethanol or drug abuse, 1 point: drug predisposing to bleeding, 1 point. $A C E$ angiotensin converting enzyme, $A F$ atrial fibrillation, $A R B$ angiotensin II receptor blocker, $C C B$ calcium channel blocker, $C O P D$ chronic obstructive pulmonary disease, $O A C$ oral anticoagulant, $S M D$ standardized mean difference, TIA transient ischemic attack.

\begin{tabular}{|c|c|c|c|c|c|c|c|c|c|}
\hline & $\begin{array}{l}\text { Number of } \\
\text { events }\end{array}$ & Person years & $\begin{array}{l}\text { Event rate (100 } \\
\text { person-years) }\end{array}$ & $\begin{array}{l}\text { Number of } \\
\text { events }\end{array}$ & Person years & $\begin{array}{l}\text { Event rate (100 } \\
\text { person-years) }\end{array}$ & $\begin{array}{l}\text { Absolute } \\
\text { reduction in } \\
\text { event rate (95\% } \\
\text { CI) }\end{array}$ & $\begin{array}{l}\text { Hazard ratio } \\
(95 \% \text { CI })^{*}\end{array}$ & $p$ value \\
\hline \multicolumn{10}{|c|}{ Ablation vs. medical therapy } \\
\hline & \multicolumn{3}{|c|}{ Medical Therapy $(\mathrm{N}=12,058)$} & \multicolumn{3}{|c|}{ Ablation $(\mathrm{N}=3173)$} & & & \\
\hline All-cause death & 3940 & 63,486 & 6.2 & 394 & 14,155 & 2.8 & $3.4(3.0-3.9)$ & $0.42(0.27-0.65)$ & $<0.001$ \\
\hline $\begin{array}{l}\text { Cardiovascular } \\
\text { death }\end{array}$ & 1912 & 63,486 & 3.0 & 174 & 14,155 & 1.2 & $1.8(1.5-2.1)$ & $0.38(0.22-0.62)$ & $<0.001$ \\
\hline Heart failure & 2540 & 54,200 & 4.7 & 273 & 12,937 & 2.1 & $2.6(2.2-3.0)$ & $0.39(0.33-0.46)$ & $<0.001$ \\
\hline Stroke/SE & 2057 & 56,534 & 3.6 & 301 & 13,130 & 2.3 & $1.3(1.0-1.7)$ & $0.44(0.37-0.53)$ & $<0.001$ \\
\hline $\begin{array}{l}\text { Sudden cardiac } \\
\text { death }\end{array}$ & 601 & 62,868 & 1.0 & 65 & 14,064 & 0.5 & $0.5(0.3-0.7)$ & $0.47(0.19-1.18)$ & 0.108 \\
\hline \multicolumn{10}{|c|}{ Ablation vs. AAD treated } \\
\hline & \multicolumn{3}{|c|}{ AAD treated $(\mathrm{N}=7976)$} & \multicolumn{3}{|c|}{ Ablation $(\mathrm{N}=3173)$} & & & \\
\hline All-cause death & 2047 & 42,158 & 4.9 & 345 & 14,352 & 2.4 & $2.5(2.1-2.8)$ & $0.49(0.34-0.71)$ & $<0.001$ \\
\hline $\begin{array}{l}\text { Cardiovascular } \\
\text { death }\end{array}$ & 977 & 42,158 & 2.3 & 153 & 14,352 & 1.1 & $1.3(1.0-1.5)$ & $0.43(0.27-0.68)$ & $<0.001$ \\
\hline Heart failure & 1574 & 36,520 & 4.3 & 256 & 13,171 & 1.9 & $2.4(2.0-2.7)$ & $0.35(0.30-0.42)$ & $<0.001$ \\
\hline Stroke/SE & 1222 & 38,261 & 3.2 & 281 & 13,370 & 2.1 & $1.1(0.8-1.4)$ & $0.45(0.37-0.54)$ & 0.004 \\
\hline $\begin{array}{l}\text { Sudden cardiac } \\
\text { death }\end{array}$ & 340 & 41,716 & 0.8 & 58 & 14,258 & 0.4 & $0.4(0.2-0.6)$ & $0.49(0.22-1.08)$ & 0.078 \\
\hline \multicolumn{10}{|c|}{ Ablation vs. rate control only } \\
\hline & \multicolumn{3}{|c|}{ Rate control only $(\mathrm{N}=4082)$} & \multicolumn{3}{|c|}{ Ablation $(\mathrm{N}=3173)$} & & & \\
\hline All-cause death & 1916 & 30,188 & 6.3 & 342 & 14,415 & 2.4 & $4.0(3.5-4.4)$ & $0.39(0.27-0.57)$ & $<0.001$ \\
\hline $\begin{array}{l}\text { Cardiovascular } \\
\text { death }\end{array}$ & 933 & 30,188 & 3.1 & 156 & 14,415 & 1.1 & $2.0(1.7-2.3)$ & $0.35(0.22-0.56)$ & $<0.001$ \\
\hline Heart failure & 1180 & 26,143 & 4.5 & 253 & 13,279 & 1.9 & $2.6(2.2-3.0)$ & $0.36(0.30-0.44)$ & $<0.001$ \\
\hline Stroke/SE & 1055 & 26,492 & 4.0 & 286 & 13,415 & 2.1 & $1.9(1.5-2.2)$ & $0.37(0.30-0.45)$ & $<0.001$ \\
\hline $\begin{array}{l}\text { Sudden cardiac } \\
\text { death }\end{array}$ & 289 & 29,896 & 1.0 & 53 & 14,329 & 0.4 & $0.6(0.4-0.8)$ & $0.43(0.21-0.88)$ & 0.020 \\
\hline
\end{tabular}

Table 2. Risk of clinical outcomes in propensity score-weighted patients stratified by treatment. ${ }^{*}$ Adjusted for age, sex, income, $\mathrm{AF}$ duration, $\mathrm{CHA}_{2} \mathrm{DS}_{2}$-VASc score, modified HAS-BLED score, hospital frailty risk score, Charlson comorbidity index, hypertension, diabetes, ischemic stroke/TIA, myocardial infarction, peripheral arterial disease, hypertrophic cardiomyopathy, chronic kidney disease, end stage renal disease, liver disease, malignancy, hyperthyroidism, hypothyroidism, venous thromboembolism, COPD, intracranial bleeding, previous cardioversion, history of bleeding, baseline use of warfarin, non-vitamin K antagonist oral anticoagulant, aspirin, clopidogrel, beta-blocker, ACE-inhibitor/ARB, dihydropyridine/nondihydropyridine $\mathrm{CCB}$, statin, diuretics, digoxin, and OAC coverage rate of time at risk. $A A D$ antiarrhythmic drug, $C I$ confidence interval, SE systemic embolism. Other abbreviations are same as Table 1.

elderly ( $\geq 75$ years) and optimally anticoagulated (proportion of days covered by anticoagulant $\geq 80 \%$ ) patients (Fig. 3).

The risk of cardiovascular death was $62 \%$ lower (1.2 and 3.0 per 100 person-years, respectively; HR 0.38 , 95\% CI $0.32-0.62, p<0.001$ ), and the cumulative incidence of cardiovascular death was significantly lower in patients with AF ablation compared to those with medical therapy $(p<0.001)$ (Table 2, Fig. 2B). The risk of cardiovascular mortality was also reduced in patients with AF ablation compared to those treated with AAD (HR 0.43, 95\% CI $0.27-0.68, p<0.001$ ) and rate control only (HR 0.35, 95\% CI $0.22-0.56, p<0.001$ ) (Table 2). Subgroup analyses about the risk of cardiovascular death showed that it was reduced in most subgroups except in heart failure patients without hypertension and those with vascular disease (Fig. 4). 
A

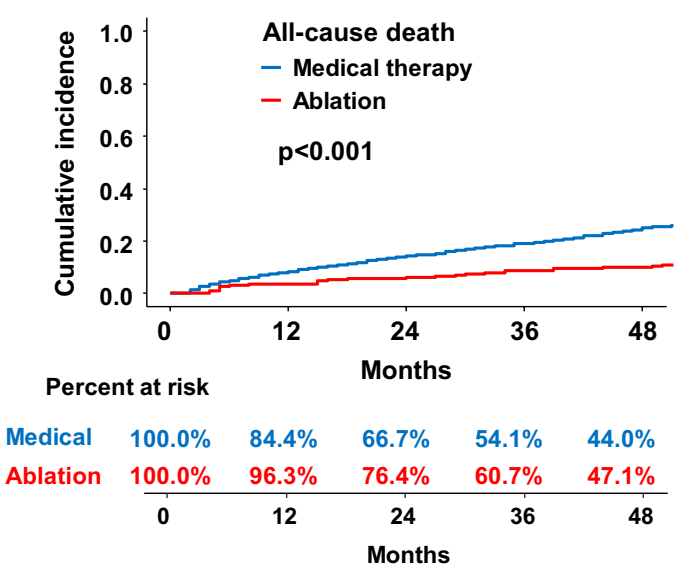

B

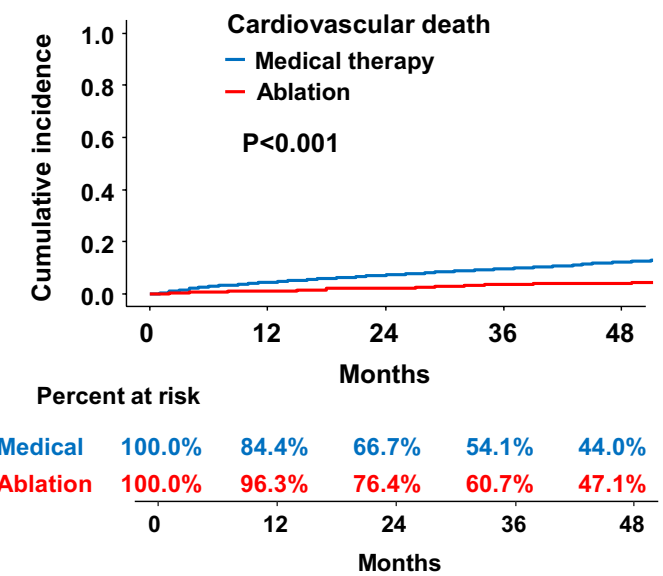

Figure 2. Weighted cumulative incidence curves of (A) all-cause death and (B) cardiovascular death for ablated and medical therapy patients. Figure prepared in R software (version 3.6.1, R Core Team, 2019, Vienna, Austria $)^{22}$.

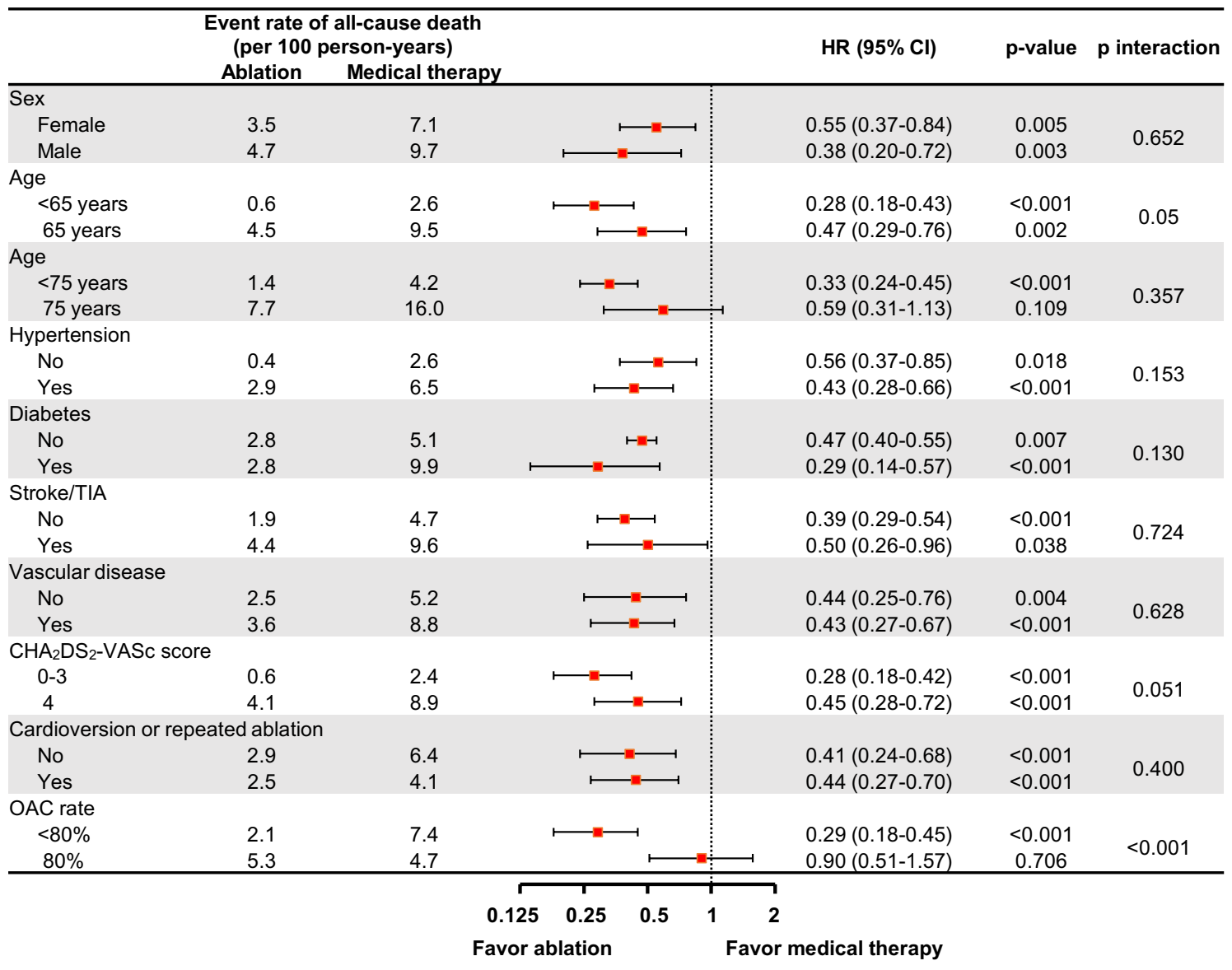

Figure 3. Subgroup analyses of the risk of all-cause death. HR: hazard ratio, TIA: transient ischemic attack, OAC: oral anticoagulant.

Reduced heart failure admission and Stroke/SE in ablated patients. AF ablation in heart failure patients was related to lower incidence and risk of heart failure admission (2.1 and 4.7 per 100 person-years, respectively; HR $0.39,95 \%$ CI $0.33-0.46, p<0.001)$ and stroke/SE (2.3 and 3.6 per 100 person-years, respectively; HR $0.44,95 \%$ CI $0.37-0.53, p<0.001$ ) compared to the medical therapy (Table 2 ). The cumulative incidence of 


\begin{tabular}{|c|c|c|c|c|c|c|}
\hline & \multicolumn{2}{|c|}{$\begin{array}{l}\text { Event rate of CV death } \\
\text { (per } 100 \text { person-years) }\end{array}$} & & \multirow[t]{2}{*}{ HR (95\% Cl) } & \multirow[t]{2}{*}{ p-value } & \multirow[t]{2}{*}{$p$ interaction } \\
\hline & Ablation & No ablation & & & & \\
\hline \multicolumn{7}{|l|}{$\overline{\text { Sex }}$} \\
\hline Female & 1.9 & 3.8 & 一—— & $0.55(0.32-0.95)$ & 0.032 & \multirow{2}{*}{0.067} \\
\hline Male & 0.9 & 2.5 & $\longmapsto$ & $0.26(0.12-0.57)$ & $<0.001$ & \\
\hline \multicolumn{7}{|l|}{ Age } \\
\hline$<65$ years & 0.3 & 1.4 & $\longmapsto$ & $0.25(0.12-0.49)$ & $<0.001$ & \multirow{2}{*}{0.177} \\
\hline 65 years & 2.0 & 4.5 & $\longmapsto$ & $0.41(0.23-0.72)$ & 0.002 & \\
\hline \multicolumn{7}{|l|}{ Age } \\
\hline$<75$ years & 0.6 & 2.1 & $\longmapsto \square$ & $0.29(0.17-0.48)$ & $<0.001$ & \multirow[b]{2}{*}{0.388} \\
\hline 75 years & 3.4 & 7.7 & ——- & $0.49(0.24-0.96)$ & 0.048 & \\
\hline \multicolumn{7}{|l|}{ Hypertension } \\
\hline No & 0.1 & 1.0 & $\longmapsto$ & $0.61(0.21-1.62)$ & 0.128 & \multirow{2}{*}{0.901} \\
\hline Yes & 1.3 & 3.2 & $\longmapsto$ & $0.37(0.22-0.62)$ & $<0.001$ & \\
\hline \multicolumn{7}{|l|}{ Diabetes } \\
\hline No & 1.0 & 2.4 & —— & $0.43(0.27-0.66)$ & $<0.001$ & \multirow{2}{*}{0.841} \\
\hline Yes & 1.8 & 4.9 & $\longrightarrow$ & $0.33(0.13-0.85)$ & 0.021 & \\
\hline \multicolumn{7}{|l|}{ Stroke/TIA } \\
\hline No & 0.9 & 2.2 & —— & $0.41(0.26-0.65)$ & $<0.001$ & \multirow[b]{2}{*}{0.800} \\
\hline Yes & 1.8 & 4.8 & 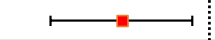 & $0.38(0.17-0.83)$ & 0.015 & \\
\hline \multicolumn{7}{|c|}{ Vascular disease } \\
\hline No & 0.6 & 2.4 & $\longrightarrow$ & $0.21(0.09-0.48)$ & $<0.001$ & \multirow[b]{2}{*}{0.057} \\
\hline Yes & 2.8 & 4.4 & $\longmapsto-$ & $0.67(0.39-1.14)$ & 0.137 & \\
\hline \multicolumn{7}{|c|}{$\mathrm{CHA}_{2} \mathrm{DS}_{2}$-VASc score } \\
\hline $0-3$ & 0.2 & 1.1 & $\longrightarrow$ & $0.19(0.09-0.43)$ & $<0.001$ & \multirow{2}{*}{0.059} \\
\hline 4 & 1.8 & 4.4 & $\longmapsto$ & $0.39(0.23-0.67)$ & $<0.001$ & \\
\hline \multicolumn{7}{|c|}{ Cardioversion or repeated ablation } \\
\hline No & 1.4 & 1.3 & $\longmapsto-$ & $0.40(0.22-0.73)$ & 0.003 & \multirow{2}{*}{0.250} \\
\hline Yes & 0.7 & 2.7 & $\longmapsto=$ & $0.21(0.11-0.38)$ & $<0.001$ & \\
\hline \multicolumn{7}{|l|}{ OAC rate } \\
\hline$<80 \%$ & 1.2 & 3.5 & $\longmapsto$ & $0.34(0.18-0.63)$ & $<0.001$ & \multirow[b]{2}{*}{0.307} \\
\hline $80 \%$ & 1.3 & 2.4 & $\longmapsto \square$ & $0.45(0.24-0.84)$ & 0.012 & \\
\hline
\end{tabular}

Figure 4. Subgroup analyses of the risk of cardiovascular death. HR, hazard ratio, TIA, transient ischemic attack, OAC, oral anticoagulant.
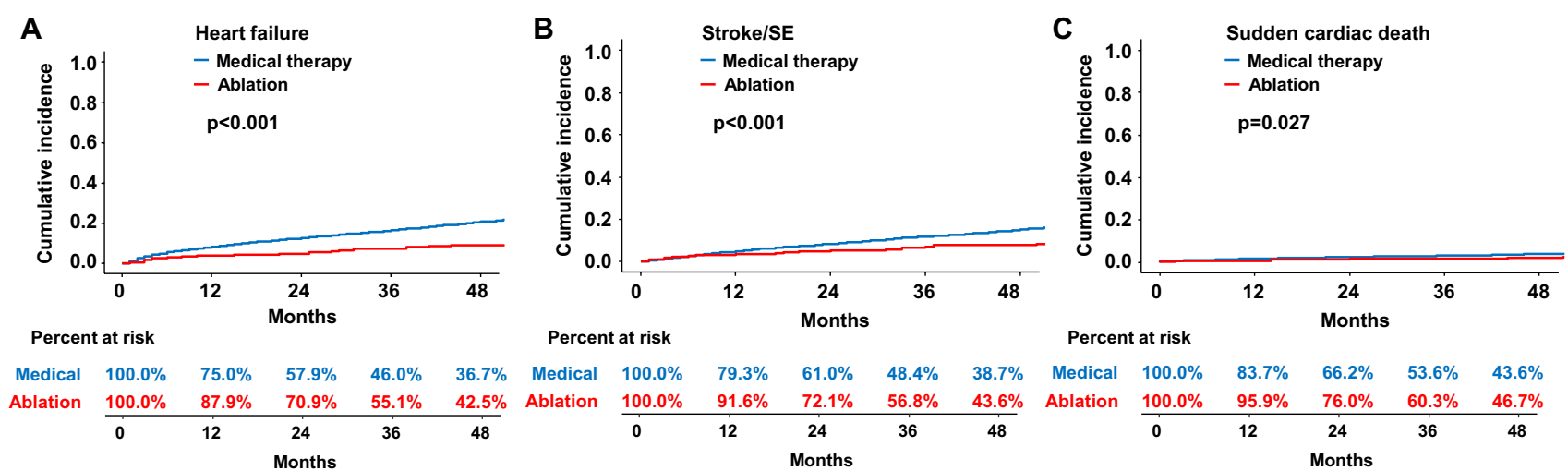

Figure 5. Weighted cumulative incidence curves of $(\mathbf{A})$ heart failure, $(\mathbf{B})$ ischemic stroke/SE, and (C) sudden cardiac death for patients with ablation or medical therapy. SE, systemic embolism. Figure prepared in $\mathrm{R}$ software (version 3.6.1, R Core Team, 2019, Vienna, Austria) ${ }^{22}$.

heart failure admission ( $p<0.001$, Fig. 5A) and stroke/SE $(p<0.001$, Fig. 5B) in patients with AF ablation was significantly lower than those with medical therapy.

The risk of heart failure admission in patients with AF ablation was lower than those treated with AAD (HR $0.35,95 \%$ CI $0.30-0.42, p<0.001$ ) and rate control only (HR $0.36,95 \%$ CI $0.30-0.44, p<0.001$ ). AF ablation was also associated with a lower risk of ischemic stroke/SE compared to AAD treatment (HR 0.45, 95\% CI 0.37-0.54, $p<0.001$ ) and rate control only (HR 0.37, 95\% CI 0.30-0.45, $p<0.001$ ) (Table 2).

However, the risk of sudden cardiac death of ablated patients was lower than rate control only patients, but not medical therapy or AAD treated patients (Table 2, Fig. 5C). 
Sensitivity analyses. First, the results using one-to-one propensity score matching (instead of propensity score weighting) were consistent with the primary results. HR for all-cause death when AF ablation was performed was 0.38 (95\% CI $0.31-0.47, p<0.001)$ compared to medical therapy, 0.41 (95\% CI $0.34-0.51, p<0.001$ ) compared to AAD treatment, and 0.59 (95\% CI $0.45-0.77, p<0.001)$ compared to rate control only. In addition, AF catheter ablation was associated with a lower risk of cardiovascular death, admission for heart failure, and ischemic stroke/SE compared to medical therapy, AAD treatment, and rate control in 1:1 propensity score matching analysis (Supplementary Table S5). Second, a comparison of the group of patients with heart failure who underwent AF ablation and the contemporary matched group of heart failure patients without a history of AF is shown in Supplementary Table S6. Compared with propensity score-weighted heart failure patients without a history of AF, the risks of all-cause mortality, admission for heart failure, and ischemic stroke/SE were not significantly higher in heart failure patients who underwent AF ablation. Third, AF ablation had no significant relationship with any of the falsification endpoints (Supplementary Table S7).

\section{Discussion}

The main finding of this study was that in real-world data, ablation for AF in patients with heart failure was associated with a significantly lower risk of mortality than medical therapy. Second, AF patients with heart failure who underwent ablation had a lower risk of hospitalization for heart failure and stroke/SE than the non-ablated matched patients. However, ablation was not associated with the reduced risk of death in the elderly ( $\geq 75$ years) and optimally anticoagulated patients. This finding supports the beneficial effect of AF catheter ablation in realworld AF patients with heart failure.

The effect of ablation on mortality and heart failure admission. Complication rates related to the catheter ablation procedure may be higher in patients with heart failure compared with general cohorts of patients undergoing an AF ablation. In the CASTLE-AF (Catheter Ablation versus Standard Conventional Therapy in Patients with Left Ventricular Dysfunction and Atrial Fibrillation) study, procedure-related complications or serious adverse events occurred in $7.8 \%{ }^{15}$. In the meta-analysis of patients with heart failure, the peri-procedural major complication rate of AF catheter ablation was $6.3 \%{ }^{24}$, while the complication rate in a contemporary cohort of general patients undergoing AF ablation was $2.3 \%{ }^{25}$. However, consistently in previous studies of heart failure patients, AF catheter ablation improved left ventricular systolic function and reduced adverse outcomes including readmission due to heart failure ${ }^{12-14}$. A randomized control study, the CASTLE-AF trial, showed that catheter ablation for AF in patients with heart failure was associated with a significantly lower rate of a composite endpoint of death from any cause or hospitalization for worsening heart failure than medical therapy. Ablation lowered the risk of death with an HR of $0.53(95 \% \text { CI } 0.32-0.86, p=0.01)^{15}$. In the current study, the risk reduction of death in the ablated group compared to the non-ablated group was very similar to the improvement in outcome for all-cause mortality in the CASTLE-AF trial.

In contrast to the current study and the CASTLE-AF trial, the AMICA (the Atrial Fibrillation Management in Congestive Heart Failure With Ablation) trial, a randomized controlled trial published in 2019, failed to show a significant benefit of catheter ablation over best medical therapy ${ }^{26}$. However, the AMICA trial included only patients with persistent/longstanding persistent AF and heart failure with severely reduced left ventricular ejection fraction (LVEF) less than 35\%. Our study included not only persistent AF patients but also paroxysmal AF patients. Heart failure patients with preserved LVEF also might be included because heart failure was defined through the diagnosis code of the administrative database. The limited benefit of catheter ablation in the AMICA trial can be explained because study patients were generally sicker and with more advanced heart failure compared with the patient in our study. Ablation therapy might have limited benefit over medical treatment in patients with seriously advanced heart failure despite achieving a lower AF burden. It should be important to select the patient carefully to perform catheter ablation to maximize the benefit of catheter ablation.

In the subgroup analysis of this study, all-cause mortality was not significantly reduced by ablation in the elderly population and those with strict anticoagulation. Attenuation of the benefits by ablation in the elderly population can also be found in the subgroup analysis of CASTLE-AF and CABANA trial ${ }^{15,16}$. The more advanced left atrial remodeling of older patients and the consequent decrease in AF ablation efficiency may be the cause, but this remains unproven. Anticoagulation therapy (vitamin $\mathrm{K}$ antagonists) has been shown to reduce overall mortality in individuals with AF compared with placebo when all studies are considered together (relative risk, $0.74 ; 95 \%$ CI, $0.57-0.97)^{27}$. No significant reduction of the outcome by ablation in patients with strict anticoagulation emphasizes that effective anticoagulation is the most important to reduce mortality in AF patients with heart failure. Catheter ablation for maintaining sinus rhythm is the next important step. The current study suggests that ablation might be related to the reduction of cardiovascular mortality in real-world AF patients with heart failure.

The effect of ablation on other outcomes. Ablation also reduced the risk of heart failure hospitalization in the CASTLE-AF trial and this study ${ }^{15}$. This result highlights the importance of preserving or restoring the atrial contribution to cardiac hemodynamics, as the AF catheter ablation rhythm control strategy provides additional benefits over simple control of rapid ventricular rate in patients with heart failure in AF.

The number of events of ischemic stroke was too small to have enough power to prove the benefit of ablation in the prevention of ischemic stroke in the CASTLE-AF study ${ }^{15}$. Several non-randomized observational studies have reported positive outcomes, including reduced incidence of ischemic stroke and reduced mortality in patients with AF ablation ${ }^{28-31}$. But, these favorable studies of reducing stroke after ablation have not been performed in specific patients with AF and heart failure. In the current study of AF patients with heart failure, the risks of ischemic stroke/SE was lower in patients who underwent AF ablation than those with medical therapy. $\mathrm{AF}$ and heart failure form a vicious circle with each other. Concomitant AF and heart failure synergistically 
increase the risk of stroke. Because ablation is an effective treatment for rhythm control that breaks the vicious cycle between $\mathrm{AF}$ and heart failure, it can be beneficial in stroke prevention of patients with $\mathrm{AF}$ and heart failure.

Study limitations. There are several limitations to this study. First, because the administrative database is used, it might be susceptible to errors caused by inaccuracies in coding for diagnosis. To minimize these errors, we applied definitions already validated in previous studies using the Korean NHIS data ${ }^{3-5}$. Second, a retrospective registry study like ours cannot establish a causal relationship, only the association can be reported. The propensity score weighting was used to match the two groups as closely as possible, but unknown confounding factors cannot be resolved. Third, because information on the degree of left ventricular function was unavailable, the severity of heart failure was not evaluated. Because the healthier people are more likely to receive ablation as the Supplement Table S2 shows, the heart failure severity could be different between the ablated group and the medical therapy group, which might make bias in the results. Our comparisons between ablation and medical therapy should be interpreted carefully. Fourth, Some study patients had a history of hyperthyroidism, hypothyroidism, malignancy, chronic obstructive pulmonary disease, or liver disease that may affect outcomes. Therefore, there may be concerns that the benefit of ablation in AF patients with heart failure may only be applied to a specific subgroup. Fifth, the utilization rate of oral anticoagulants in this study was lower than in other studies. It is well known that the use of oral anticoagulants in Asia-Pacific countries is low compared to other regions ${ }^{5,32}$. The utilization rate of oral anticoagulants has improved with the introduction of non-vitamin $\mathrm{K}$ antagonist oral anticoagulants (NOACs). However, in Korea, the use of NOACs was increased from 2015 as NOACs were fully reimbursed by the national insurance system. The inclusion period of our study was from 2005 to 2015, before NOAC was fully used. So oral anticoagulants were underused in this study population and a large proportion of oral anticoagulants was warfarin. The underuse of oral anticoagulants and a large proportion of warfarin among oral anticoagulants may have influenced the results of this study. Sixth, the dose and label adherence of NOACs was not evaluated. And we also did not have access to information on time-in-therapeutic range in patients using warfarin. Lack of information about the quality of oral anticoagulants treatment might interfere with results, especially the outcome of ischemic stroke and systemic embolism. Finally, to indirectly assess the relationship between successful rhythm control by ablation and all-cause and cardiovascular death, subgroup analysis was performed according to "Cardioversion or repeated ablation" as an indicator of AF recurrence after ablation. However, the exact relationships between the primary outcome or mortality and rhythm control statuses such as sinus rhythm maintenance or AF burden were not evaluated. Moreover, since there is no continuous rhythm monitoring data, the subclinical or asymptomatic AF recurrence can not be assessed.

\section{Conclusion}

Ablation may be associated with lower incidences of death, heart failure admission, and stroke/SE in real-world $\mathrm{AF}$ patients with heart failure, supporting the role of AF ablation in heart failure.

Received: 28 March 2020; Accepted: 27 January 2021

Published online: 25 February 2021

\section{References}

1. Levy, D. et al. Long-term trends in the incidence of and survival with heart failure. N. Engl. J. Med. 347, 1397-1402. https://doi. org/10.1056/NEJMoa020265 (2002).

2. Berry, C., Murdoch, D. R. \& McMurray, J. J. Economics of chronic heart failure. Eur. J. Heart Fail. 3, 283-291. https://doi. org/10.1016/s1388-9842(01)00123-4 (2001).

3. Kim, D. et al. 10-year nationwide trends of the incidence, prevalence, and adverse outcomes of non-valvular atrial fibrillation nationwide health insurance data covering the entire Korean population. Am. Heart J. 202, 20-26. https://doi.org/10.1016/j. ahj.2018.04.017 (2018).

4. Kim, D. et al. Increasing trends in hospital care burden of atrial fibrillation in Korea, 2006 through 2015. Heart 104, $2010-2017$. https://doi.org/10.1136/heartjnl-2017-312930 (2018).

5. Lee, H. et al. The trends of atrial fibrillation-related hospital visit and cost, treatment pattern and mortality in Korea: 10 -year nationwide sample cohort data. Korean Circ. J. 47, 56-64. https://doi.org/10.4070/kcj.2016.0045 (2017).

6. Dries, D. L. et al. Atrial fibrillation is associated with an increased risk for mortality and heart failure progression in patients with asymptomatic and symptomatic left ventricular systolic dysfunction: a retrospective analysis of the SOLVD trials. Studies of left ventricular dysfunction. J. Am. Coll. Cardiol. 32, 695-703. https://doi.org/10.1016/s0735-1097(98)00297-6 (1998).

7. Wang, T. J. et al. Temporal relations of atrial fibrillation and congestive heart failure and their joint influence on mortality: the Framingham Heart Study. Circulation 107, 2920-2925. https://doi.org/10.1161/01.CIR.0000072767.89944.6E (2003).

8. Daoud, E. G. et al. Effect of an irregular ventricular rhythm on cardiac output. Am. J. Cardiol. 78, 1433-1436. https://doi. org/10.1016/s0002-9149(97)89297-1 (1996).

9. Clark, D. M., Plumb, V. J., Epstein, A. E. \& Kay, G. N. Hemodynamic effects of an irregular sequence of ventricular cycle lengths during atrial fibrillation. J. Am. Coll. Cardiol. 30, 1039-1045. https://doi.org/10.1016/s0735-1097(97)00254-4 (1997).

10. Wilber, D. J. et al. Comparison of antiarrhythmic drug therapy and radiofrequency catheter ablation in patients with paroxysmal atrial fibrillation: a randomized controlled trial. JAMA 303, 333-340. https://doi.org/10.1001/jama.2009.2029 (2010).

11. Mark, D. B. et al. Effect of catheter ablation vs medical therapy on quality of life among patients with atrial fibrillation: the CABANA randomized clinical trial. JAMA 321, 1275-1285. https://doi.org/10.1001/jama.2019.0692 (2019).

12. Chen, M. S. et al. Pulmonary vein isolation for the treatment of atrial fibrillation in patients with impaired systolic function. J. Am. Coll. Cardiol. 43, 1004-1009. https://doi.org/10.1016/j.jacc.2003.09.056 (2004).

13. Hsu, L. F. et al. Catheter ablation for atrial fibrillation in congestive heart failure. N. Engl. J. Med. 351, 2373-2383. https://doi. org/10.1056/NEJMoa041018 (2004).

14. Sacher, F. et al. Chronic atrial fibrillation ablation impact on endocrine and mechanical cardiac functions. Eur. Heart J. 29, 1290-1295. https://doi.org/10.1093/eurheartj/ehm577 (2008).

15. Marrouche, N. F. et al. Catheter ablation for atrial fibrillation with heart failure. N. Engl. J. Med. 378, 417-427. https://doi. org/10.1056/NEJMoa1707855 (2018). 
16. Packer, D. L. et al. Effect of catheter ablation vs antiarrhythmic drug therapy on mortality, stroke, bleeding, and cardiac arrest among patients with atrial fibrillation: the CABANA randomized clinical trial. JAMA 321, 1261-1274. https://doi.org/10.1001/ jama.2019.0693 (2019).

17. Kim, D. et al. Ideal blood pressure in patients with atrial fibrillation. J. Am. Coll. Cardiol. 72, 1233-1245. https://doi.org/10.1016/j. jacc.2018.05.076 (2018).

18. Kim, T. H. et al. Effect of hypertension duration and blood pressure level on ischaemic stroke risk in atrial fibrillation: nationwide data covering the entire Korean population. Eur. Heart J. 40, 809-819. https://doi.org/10.1093/eurheartj/ehy877 (2019).

19. Kim, D. et al. Risk of dementia in stroke-free patients diagnosed with atrial fibrillation: data from a population-based cohort. Eur. Heart J. 40, 2313-2323. https://doi.org/10.1093/eurheartj/ehz386 (2019).

20. Fine, J. \& Gray, R. A proportional hazards model for the subdistribution of a competing risk. J. Am. Stat. Assoc. 94, 496-509 (1999).

21. Grambsch, P. \& Therneau, T. Proportional hazards tests and diagnostics based on weighted residuals. Biometrika 81, 515-526 (1994).

22. R Core Team. R: A Language and Environment for Statistical Computing. R Foundation for Statistical Computing Vienna, Austria. https://www.R-project.org/ (2019).

23. Wimmer, N. J., Resnic, F. S., Mauri, L., Matheny, M. E. \& Yeh, R. W. Comparison of transradial versus transfemoral percutaneous coronary intervention in routine practice: evidence for the importance of "falsification hypotheses" in observational studies of comparative effectiveness. J. Am. Coll. Cardiol. 62, 2147-2148. https://doi.org/10.1016/j.jacc.2013.07.036 (2013).

24. Al Halabi, S. et al. Catheter ablation for atrial fibrillation in heart failure patients: a meta-analysis of randomized controlled trials. JACC Clin. Electrophysiol. 1, 200-209. https://doi.org/10.1016/j.jacep.2015.02.018 (2015).

25. Muthalaly, R. G. et al. Temporal trends in safety and complication rates of catheter ablation for atrial fibrillation. J. Cardiovasc. Electrophysiol. 29, 854-860. https://doi.org/10.1111/jce.13484 (2018).

26. Kuck, K. H. et al. Catheter ablation versus best medical therapy in patients with persistent atrial fibrillation and congestive heart failure: the randomized AMICA trial. Circ. Arrhythm. Electrophysiol. 12, e007731. https://doi.org/10.1161/CIRCEP.119.007731 (2019).

27. Guimaraes, P. O. et al. Effect of apixaban on all-cause death in patients with atrial fibrillation: a meta-analysis based on imputed placebo effect. Cardiovasc. Drugs Ther. 31, 295-301. https://doi.org/10.1007/s10557-017-6728-z (2017).

28. Chang, C. H. et al. Effect of radiofrequency catheter ablation for atrial fibrillation on morbidity and mortality: a nationwide cohort study and propensity score analysis. Circulation. Arrhythm. Electrophysiol. 7, 76-82. https://doi.org/10.1161/CIRCEP.113.000597 (2014).

29. Bunch, T. J. et al. Patients treated with catheter ablation for atrial fibrillation have long-term rates of death, stroke, and dementia similar to patients without atrial fibrillation. J. Cardiovasc. Electrophysiol. 22, 839-845. https://doi.org/10.1111/j.1540-8167.2011.02035 .x (2011).

30. Friberg, L., Tabrizi, F. \& Englund, A. Catheter ablation for atrial fibrillation is associated with lower incidence of stroke and death: data from Swedish health registries. Eur. Heart J. 37, 2478-2487. https://doi.org/10.1093/eurheartj/ehw087 (2016).

31. Noseworthy, P. A. et al. Atrial fibrillation ablation in practice: assessing CABANA generalizability. Eur. Heart J. 40, $1257-1264$. https://doi.org/10.1093/eurheartj/ehz085 (2019).

32. Oh, S. et al. Vitamin K antagonist control in patients with atrial fibrillation in Asia compared with other regions of the world: Realworld data from the GARFIELD-AF registry. Int. J. Cardiol. 223, 543-547. https://doi.org/10.1016/j.ijcard.2016.08.236 (2016).

\section{Acknowledgements}

This study was supported by a research grant from the Korean Healthcare Technology R\&D project funded by the Ministry of Health \& Welfare (HI15C1200, HC19C0130). The National Health Information Database was provided by the NHIS of Korea. The authors would like to thank the National Health Insurance Service for cooperation.

\section{Author contributions}

P.S.Y., D.K., and B.J. designed the study, conducted data analysis, and wrote the manuscript; E.J. participated in data analysis; J.H.S., H.T.Y., T.H.K., J.S.U., J.Y.K., H.N.P., and M.H.L. participated in data collection. All authors have reviewed and approved the final version of the manuscript.

\section{Competing interests}

Dr. Boyoung Joung has served as a speaker for Bayer, BMS/Pfizer, Medtronic, and Daiichi-Sankyo and received research funds from Medtronic and Abbott. No fees have been received directly/personally. The remaining authors have nothing to declare.

\section{Additional information}

Supplementary Information The online version contains supplementary material available at https://doi. org/10.1038/s41598-021-84256-Z.

Correspondence and requests for materials should be addressed to B.J.

Reprints and permissions information is available at www.nature.com/reprints.

Publisher's note Springer Nature remains neutral with regard to jurisdictional claims in published maps and institutional affiliations.

(c) (i) Open Access This article is licensed under a Creative Commons Attribution 4.0 International License, which permits use, sharing, adaptation, distribution and reproduction in any medium or format, as long as you give appropriate credit to the original author(s) and the source, provide a link to the Creative Commons licence, and indicate if changes were made. The images or other third party material in this article are included in the article's Creative Commons licence, unless indicated otherwise in a credit line to the material. If material is not included in the article's Creative Commons licence and your intended use is not permitted by statutory regulation or exceeds the permitted use, you will need to obtain permission directly from the copyright holder. To view a copy of this licence, visit http://creativecommons.org/licenses/by/4.0/.

(C) The Author(s) 2021 